\title{
A diminished propensity to compute scalar implicatures is linked to autistic traits
}

\author{
Greta Mazzaggio \\ University of Trento \\ greta.mazzaggio@unitn.it \\ Luca Surian \\ University of Trento \\ luca.surian@unitn.it
}

\begin{abstract}
We investigated whether there is an association between autistic traits in the broader phenotype and the ability to compute scalar implicatures. Previous studies found that the frequency of autistic traits is higher in students of science than of humanities. Here we recorded the frequency of rejection of underinformative scalar items in students enrolled either in a science or in a humanities curriculum and assessed their autistic traits using the Autism-Spectrum Quotient questionnaire. We found that rejections were less frequent in science curricula students than in humanities curricula students. Moreover, rejections were associated negatively with autistic traits and positively with performance on Theoryof-Mind tasks. These findings suggest that autism cognitive phenotype is negatively associated with a propensity to spontaneously derive scalar implicatures.
\end{abstract}

Keywords: scalar implicatures; quantifiers; Autism-Spectrum Quotient; pragmatics; Theory of Mind

\section{Introduction}

Grice (1975) made an important distinction between saying and conveying, which corresponds to a distinction between utterance meaning (i.e., the semantic meaning) and speaker meaning. An example is given in (1).

(1) A: Do you want to come out for a drink?

B: I have a job interview tomorrow.

The literal meaning of B's response (the utterance meaning) does not provide a direct answer to A's question. However, the answer is provided by the speaker meaning, it seems that B is unable to accept A's invitation. A's recognition of this meaning depends upon the specific (particular) contextual features. In a neutral context, the utterance I have a job interview 
tomorrow does not convey that the speaker is unable to go out for a drink. In this example, such information is implicated and this is why we speak about "implicatures".

Grice distinguished particularized conversational implicatures, like in (1), from generalized conversational implicatures, which are, differently from the former, context-independent. An example of generalized conversational implicatures are "scalar implicatures", which refer to sets of lexical items that constitute a scale in which the items are ordered with respect to their informativeness. The speaker's use of a weaker term in a scale implicates the negation of all the stronger terms (Horn 1972). Considering the quantifiers scale 〈some, all in (2) B's use of some implicates that he did not buy all the books for the exam, even if the semantic meaning of the utterance in $(2 \mathrm{~B})$ is that $\mathrm{B}$ bought some and possibily all the books.

(2) A: Did you buy the books for the English exam?

B: I bought some.

According to Grice (1975), addressees derive an implicature as a result of assuming that speakers adhere to a Cooperative Principle, which, in turn, requires them to obey a set of implicit rules or maxims. In the example in (2), if B bought all the books for the exam, then B's utterance violates the first Quantity maxim (i.e., make your contribution as informative as required) because the quantifier some is less informative than all. The reason for which the existential quantifier some is less informative than the universal quantifier all is because the latter can be true in less circumstances.

Indeed, as expressed in (3a), a sentence like All As are Bs can be true if, and only if (iff), all the elements present in the set of As are also present in the set of Bs. On the other hand, as expressed in (3b), a sentence like Some As are Bs is true iff the interception of As and Bs is not empty (Katsos et al. 2016). This means that for (3a) to be true, (3b) must be true as well, but (3b) can be true also if (3a) is not. In other words, the quantifier some can logically be interpreted as 'some and possibly all' but, since there is a more informative quantifier (i.e., all) available in the scale, some is interpreted pragmatically as 'some but not all'. This kind of reasoning can be applied for several other sets of terms than can be ordered in a scale, such as logical operators ( $\langle$ or, and $\rangle)$, modals (〈might, must $\rangle)$, adjectives

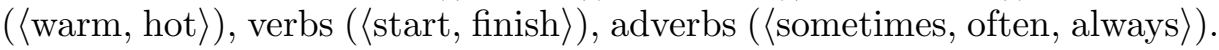

(3) a. 'All As are Bs' is true iff $A \cap B=A$

b. 'Some As are Bs' is true iff $A \cap B \neq \emptyset$ 
Two main theoretical views on the computation of scalar implicatures have been proposed (for a review of all the models see Chemla \& Singh 2014a). An ongoing debate concerns whether scalar implicatures are computed through linguistic (e.g., semantic or syntactic) or pragmatic mechanisms that require mental state reasoning. For example, Chierchia's (2013) Extended Standard Gricean Theory assumes that a scalar implicature is derived from an utterance such as (4a) by means of an exhaustivity operator $(O)$, which is a covert counterpart of only as shown in (4b). The result of the operator is shown in (4c). The "exhaustification process" operates on the set of lexical scalar alternatives when they are relevant to the conversational goals. If deriving the implicature is not advantageous in a particular context, the exhaustification process will not occur.

(4) a. Some linguists are smart.

b. Only some linguists are smart.

c. $O$ [some linguists are smart].

As we have previously seen, other researchers attribute the computation of scalar-implicatures to the ability to recognize the speaker's communicative intentions. Based on Grice's account, if we consider the dialogue in (2), the interlocutor A, after listening the sentence I bought some uttered by B, should take into consideration the alternative sentence I bought all that B could have uttered instead. Because A knows (and knows that B knows too) that some is less informative than all and that $\mathrm{B}$ used some instead of all, A assumes that if B bought all the books, then B would have used the quantifier all as required by the Quantity maxim. This ability to "read the mind" of another person is known in the literature as Theory of Mind (Premack \& Woodruff 1978) or mentalizing ability (Frith et al. 1991). Chemla and Singh (2014a) suggested that both the Gricean view and the grammatical theory (i.e., Extended Standard Gricean Theory), theorize the involvement, at a certain point, of a pragmatic decision in scalar-implicature computation. However, there is no consensus on the role of Theory of Mind in pragmatic inferences. While some authors support the idea of a central role of mentalizing abilities (Nieuwland et al. 2010; Surian et al. 1996), other authors do not (Andrés-Roqueta \& Katsos 2017; Antoniou et al. 2016). Studies on population with less mature Theory-of-Mind skills might be of interest for this specific theoretic aspect of the debate.

People with Autism Spectrum Disorder (ASD) present impairment in Theory-of-Mind development (Baron-Cohen et al. 1985) and this lack of or non-mature Theory of Mind has often been linked to pragmatic deficits 
(Baron-Cohen 1988). ASD population is well known for presenting pragmatic difficulties, for example with obeying Gricean Maxims (Capps et al. 1998; Surian et al. 1996), with comprehending jokes (Baron-Cohen 1997b; Reddy et al. 2002), irony and metaphors (Happé 1993; MacKay \& Shaw 2004). A recent study reported that adolescents with ASD can compute scalar implicatures, but they seem do so without reasoning about the interlocutor's epistemic states (Hochstein et al. 2018).

The aim of the present study was to further investigate scalar-implicature computation and the role of epistemic reasoning by testing typically developing adult students enrolled in scientific and humanistic curricula (Experiment 1) and by assessing the presence of autistic traits (Experiment 2). Many previous works by Baron-Cohen and colleagues found that autistic traits, such as weak Theory-of-Mind skills, are associated both to gender, with males scoring higher than females, and to vocational choices, with peoples involved in some scientific or technical profession scoring higher than people involved in social professions (Baron-Cohen 1997a; Baron-Cohen et al. 1997; Baron-Cohen 1998; Baron-Cohen et al. 2001). If scalar-implicature computation is linked to mental state reasoning, then one should expect less pragmatic answers and more logic interpretations of underinformative sentences (i.e., sentences including some) in students of scientific curricula and in males, than in students of humanities curricula and in females.

Baron-Cohen (1997a) proposed that people with high-functioning autism have weak "folk psychology" abilities, such as inferring mental states from people's behavior, but well developed "folk physics" abilities, such as inferring the physical causes of natural events. These folk physics skills are superior compared to typical population (Baron-Cohen et al. 1986; Sigman et al. 1987; Baron-Cohen 1989; Leekam \& Perner 1991; Leslie \& Thaiss 1992; Jolliffe \& Baron-Cohen 1997).

Coherently with such proposal, Baron-Cohen et al. (1997) found that fathers and grandfathers of children with autism are more likely to be employed in fields such engineering and informatics than fathers and grandfathers of children without autism. In line with this, Baron-Cohen (1998) showed that students of physics, engineering and mathematics have more biological relatives with autism compared to students of literature. BaronCohen et al. (2001) also found that male students and scientists score higher on autistic traits compared to females and to students of humanities and social sciences.

Thus, Experiment 2 included the "Autism-spectrum Quotient", which is a standardized tool to measure the extent of autistic traits in the typ- 
ical adult population with an average IQ (Baron-Cohen et al. 2001). We were particularly interested in the relation between scalar-implicature computation and autistic traits measured by the Autism-spectrum Quotient, such as social skills, attention switching skills, attention to detail skills, communication skills, imagination skills and, as we proposed, Theory-ofMind skills.

A strong difference in autistic traits, when comparing two groups, is of course found when one compares a group of people with autism with a control group of people without such disorder. In our case in which autistic traits in the broader phenotype are assessed, if we assume that the computation of scalar implicatures involves some reasoning about mental states and this type of reasoning is negatively associated with the presence of autistic traits, then we should predict that the amount of pragmatic responses obtained in a scalar-implicature task would be a function of autistic traits. Moreover, since these traits are also associated with different vocational choices, we should also predict that pragmatic responses in the scalar-implicature task would vary as a function of the course attended by students. To sum up, with our study we want to address the debate around the role of Theory of Mind in the computation of scalar implicatures and we can also explore whether pragmatic inferences in typical adults might be influenced by particular traits in their personality.

\section{Experiment 1}

\subsection{Method}

\subsubsection{Participants}

Participants were 428 students (277 females, mean age 23.2 years, $S D=$ 4.6) who were attending Italian universities. Students were recruited online in groups for students willing to exchange informations and received no compensation in exchange for participation. Following Baron-Cohen et al. (2001), participants were divided into a "science group" $(N=176$, 99 males, mean age $22.8, S D=3.76)$ and a "humanities group" $(N=252$, 199 females, mean age $23.5, S D=5.12)$. The science group consisted of students enrolled in mathematics $(N=74)$, chemistry $(N=4)$, medicine $(N=21)$, engineering $(N=21)$, physics $(N=19)$, informatics $(N=19)$, neuroscience $(N=8)$ and natural sciences $(N=10)$. The humanities and social sciences group consisted of students enrolled in psychology $(N=$ $201)$, sociology $(N=5)$, law $(N=6)$, literature and philosophy $(N=6)$, foreign languages and literatures $(N=8)$, economy $(N=15)$, archeology 
and architecture $(N=3)$, history $(N=5)$, and communication sciences and social services $(N=3)$. Two participants were eliminated because they were less than 18 years old. University of Trento's Ethical Committee has approved this study that is part of a more extensive research on pragmatic deficits in ASD population.

\subsubsection{Materials and procedure}

Participants completed an online Sentence Evaluation Task, that has been used in a number of previous studies of scalar implicatures (see Noveck 2001; Guasti et al. 2005; Pouscoulous et al. 2007); sentences were adapted from Bott \& Noveck (2004). The experiment ran through Google Forms. On each trial, a sentence with the basic form, All/Some $X$ are $Y$, was presented and participants indicated whether they agreed by using the mouse to click on Agree or Disagree displayed below the sentence. There were 32 sentences presented, half began with all and half began with some. Of the sentences with all, 8 were universally true ("All-true") and 8 were false or absurd statements ("All-false"). Of the sentences with some, 8 were true ("Some-true") and 8 were underinformative ("Some-underinformative"). A pseudo-randomized order has been used: one All-true, one All-false and one Some-true items had always be presented before the first Some-underinformative item in order to make the scale salient (Foppolo et al. 2012). In Table 1 some examples are presented. The complete list of sentences translated from Italian is given in Appendix 1.

Table 1: Typologies, examples and expected answers of the sentences used in Experiments 1 and 2

\begin{tabular}{lll}
\hline Type & Example sentence & Expected answer \\
\hline All-true & All snakes are reptiles & Agree \\
All-false & All animals are carnivorous & Disagree \\
Some-true & Some dogs are Labrador & Agree \\
Some-underinformative & Some children are humans & Agree (Logic); \\
& & Disagree (Pragmatic) \\
\hline
\end{tabular}

We coded 0 for wrong answers in control sentences and for pragmatic answers in underinformative sentences (disagree) and 1 for correct answers in control sentences and for logic answers in underinformative sentences (agree). The coding has been made with the use of Microsoft Excel software. 


\subsection{Results and discussion}

Agree was a correct response for the 16 true sentences (8 All-true and 8 Some-true), and Disagree was a correct response for the 8 All-false sentences. Both the science group and the humanities group were highly accurate in responding to all three sentence-types (mean correct responses range from 7.85 to 7.97 ).

Agree responses to the underinformative sentences were considered logical responses whereas Disagree responses were considered pragmatic responses. Consistent with our prediction, the number of logical responses in the science group $(M=2.91, S D=3.58)$ was significantly higher than in the humanities group $(M=2.10, S D=3.15$; Kruskal-Wallis $\mathrm{H}$ test, $\left.\chi^{2}(1)=5.29, p=.02, d=.20\right)$, as is visible in Figure $1 .^{1}$

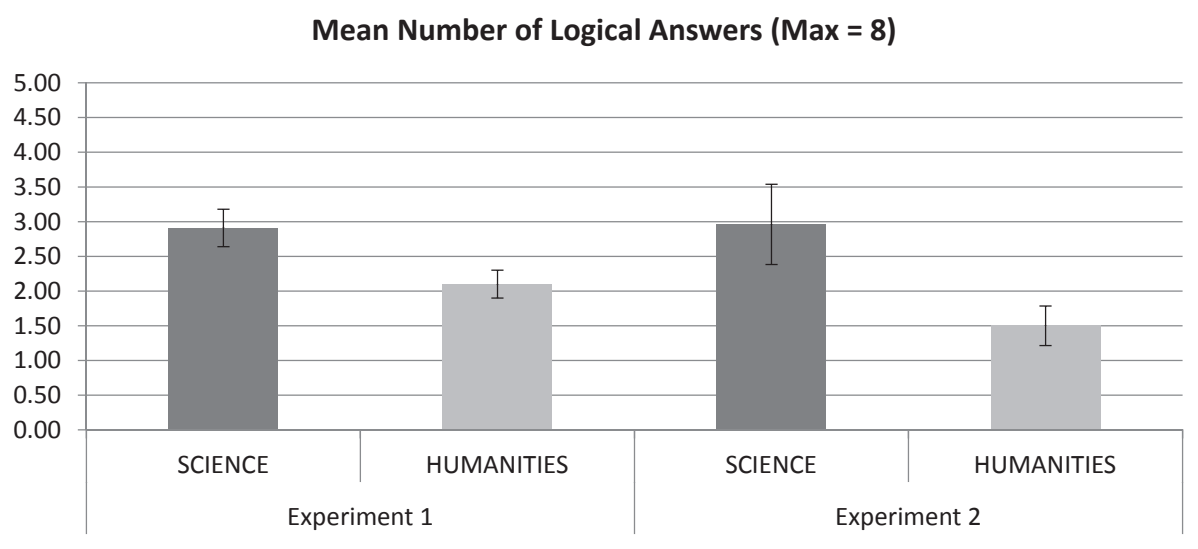

Figure 1: The Science group and Humanities group's mean number of logical responses to underinformative statements with some in Experiments 1 and 2. Error bars are standard errors of the mean.

Moreover, we found an effect of gender: the mean number of logical responses in males was $2.99(S D=3.57)$ and it was significantly higher than in females $2.14\left(S D=3.19\right.$; Kruskal-Wallis H test, $\chi^{2}(1)=9.13, p=.003$,

${ }^{1}$ As an anonymous reviewer noted, the scientific group did not answer "logically" above chance in the underinformative utterances. Actually, we never expected that typical adults might answer "logically" tout court; indeed, in the literature such behavior is characteristic of children before the age of 5 or 6 (for a short review, Chemla \& Singh 2014b, 390-391). Our prediction was that, overall, students of scientific disciplines would interpret underinformative statements in a logical way more often than students of humanities. 
$d=.28)$. However, the gender effect on the mean number of logical responses only approached significance in the science group $\left(M_{\mathrm{MALE}}=\right.$ $3.35, S D=3.72 ; M_{\text {FEMALE }}=2.37, S D=3.33$; Kruskal-Wallis $\mathrm{H}$ test, $\left.\chi^{2}(1)=3.73, p=.05, d=.25\right)$, and it was not significant in the humanities group $\left(M_{\mathrm{MALE}}=2.32, S D=3.21 ; M_{\mathrm{FEMALE}}=2.05, S D=3.14\right.$; Kruskal-Wallis H test, $\left.\chi^{2}(1)=1.96, p=.16\right)$.

\section{Experiment 2}

Experiment 2 was aimed at consolidating the results of Experiment 1 by adding a direct measure of autistic traits. Thus, Experiment 2 was identical to Experiment 1 except that participants also completed the Italian version of Baron-Cohen et al.'s (2001) Autism-spectrum Quotient questionnaire (Ruta et al. 2012).

\subsection{Method}

\subsubsection{Participants}

Participants were 198 students of different Italian universities (89 males, mean age 23.8 years, $S D=3.08$ ), who were recruited online in groups for students willing to exchange information and were not compensated for their participation. As in Experiment 1, Baron-Cohen et al.'s (2001) criteria were used to divide participants into a science group $(N=90$, 38 females, mean age 23.7 years, $S D=2.27$ ) and a humanities group $(N=108,37$ males, mean age 23.8 years, $S D=3.63)$. The science group consisted of students enrolled in mathematics $(N=38)$, physics $(N=$ $4)$, engineering $(N=27)$, medical and medical biotechonologies schools $(N=3)$ and informatics $(N=18)$. The humanities group consisted of students enrolled in literature and philosophy $(N=22)$, foreign languages and literatures $(N=35)$, communication sciences $(N=6)$, history $(N=$ $2)$, sociology $(N=2)$, art $(N=12)$, law and economy $(N=7)$, pedagogy and social sciences $(N=22)$. University of Trento's Ethical Committee has approved this study that is part of a more extensive research on pragmatic deficits in ASD population.

\subsubsection{Materials and procedure}

Experiment 2 used the materials and procedure for the online Sentence Evaluation Task as in Experiment 1, again using Google Forms. In addition, participants completed Baron-Cohen et al.'s (2001) Autism-spectrum 
Quotient before the scalar-implicature task. The measure consists of 50 questions with 10 questions assessing each of five skills/traits: social (e.g., "I prefer to do things with others rather than on my own"), attention switching (e.g., "It does not upset me if my daily routine is disturbed"), attention to detail (e.g., "I usually notice car number plates or similar strings of information"), communication (e.g., "Other people frequently tell me that what I've said is impolite, even though I think it is polite") and imagination (e.g., "If I try to imagine something, I find it very easy to create a picture in my mind").

All questions were presented with four answer choices: definitely agree, slightly agree, slightly disagree or definitely disagree. Participants had to click on one of the four answers and responses were scored as 1 or 0 . Half of the questions are created to elicit an "agree" or "slightly agree" answer, thus, with 1 point assigned if one of those two answers are given; half of the questions are created to elicit a "disagree" or "slightly disagree" answer, thus, with 1 point assigned if one of those two answers are given. The higher the obtained score, the higher the presence of autistic traits; according to Baron-Cohen et al.'s (2001), a score of $32+$ might be a cutoff to reveal the presence of clinically significant levels of autistic traits. However, obtaining a score of $32+$ does not mean the individual actually has an autistic disorder. The maximum score is 50, and Baron-Cohen et al. (2001) reported an average score of 16 for a group typical adults, an average score of 36 for a group with Asperger Syndrome or high-functioning autism (HFA), and an average score of 25 for a group of winners of the UK Mathematics Olympiad.

The coding of both tasks has been made with the use of Microsoft Excel software.

\subsection{Results and discussion}

Both the science and humanities groups were highly accurate in responding to the All-true, Some-true, and All-false statements (mean correct responses ranged from 7.68 to 7.93 ).

Like in Experiment 1, the mean number of logical answers in the science group was significantly higher than in the humanities group (Figure 1; $\mathrm{M}_{\mathrm{SC}}=2.96, S D=3.61$ vs. $M_{\mathrm{HUM}}=1.55, S D=2.67$; Kruskal-Wallis $\mathrm{H}$ test, $\left.\chi^{2}(1)=4.33, p=.04, d=.26\right)$. The difference between the males and females in the number of logical responses was not significant, $M_{\mathrm{MALE}}$ $=2.63, S D=3.46 ; M_{\text {FEMALE }}=1.83, S D=2.95$, Kruskal-Wallis $\mathrm{H}$ test, $\left.\chi^{2}(1)=2.14, p=.14\right)$. 
When looking at the AQ, we replicated Baron-Cohen et al.'s (2001) data. Figure 2 represents the means of the scores obtained in the Autismspectrum Quotient by males and females in the two groups. The science group had a higher average Autism-spectrum Quotient than the humanities group (22.84 vs. 19.33, respectively, Kruskal-Wallis $\mathrm{H}$ test, $\chi^{2}(1)=18.31, p<.001, d=.62$ ). If we consider a score of $32+$ as a cutoff for clinically significant levels of autistic traits, in our sample no students in the humanities group equaled that high score, but 6 individuals in the science group did, as it is visible in Figure 3. In addition, males had a higher average Autism-spectrum Quotient than females (22.21 vs. 19.88, respectively, Kruskal-Wallis $\mathrm{H}$ test, $\left.\chi^{2}(1)=6.81, p=.009, d=.35\right)$. Correlation between the participants' Autism-spectrum Quotient score and their number of logical responses is marginally significant (Pearson's $r=$ $0.136, N=198, p=.06)$. Such correlation, however, is also significant when considering students in the humanities group separately (Pearson $\mathrm{r}$ $=.202, N=108, p=.04$ ), but it is not significant when analyzing students of the scientific group separately (Pearson's $r=-.021, N=90, p=.85$ ).

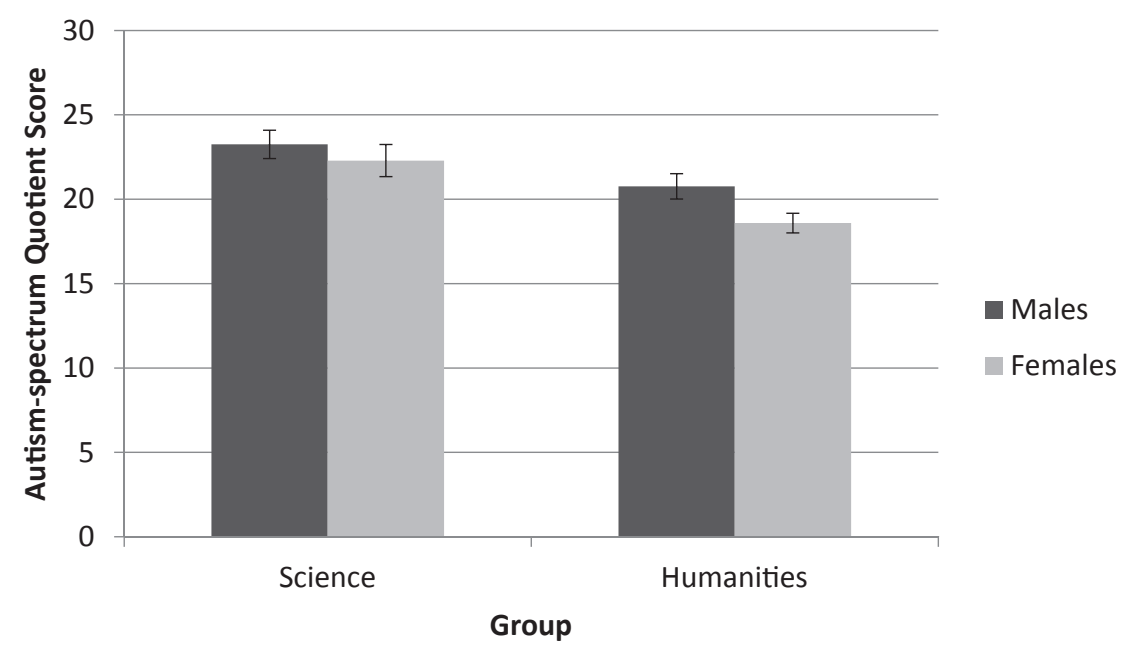

Figure 2: The mean number of scores obtained in the Autism-spectrum Quotient in Experiments 2, divided by science / humanities and males /females. Error bars are standard errors of the mean.

Thirteen items in the Autism-spectrum Quotient appeared to tap into Theory-of-Mind skills, for example, "When I'm reading a story, I find it difficult to work out the characters' intentions", "When I was young, I used to enjoy playing games involving pretending with other children", "I find it 


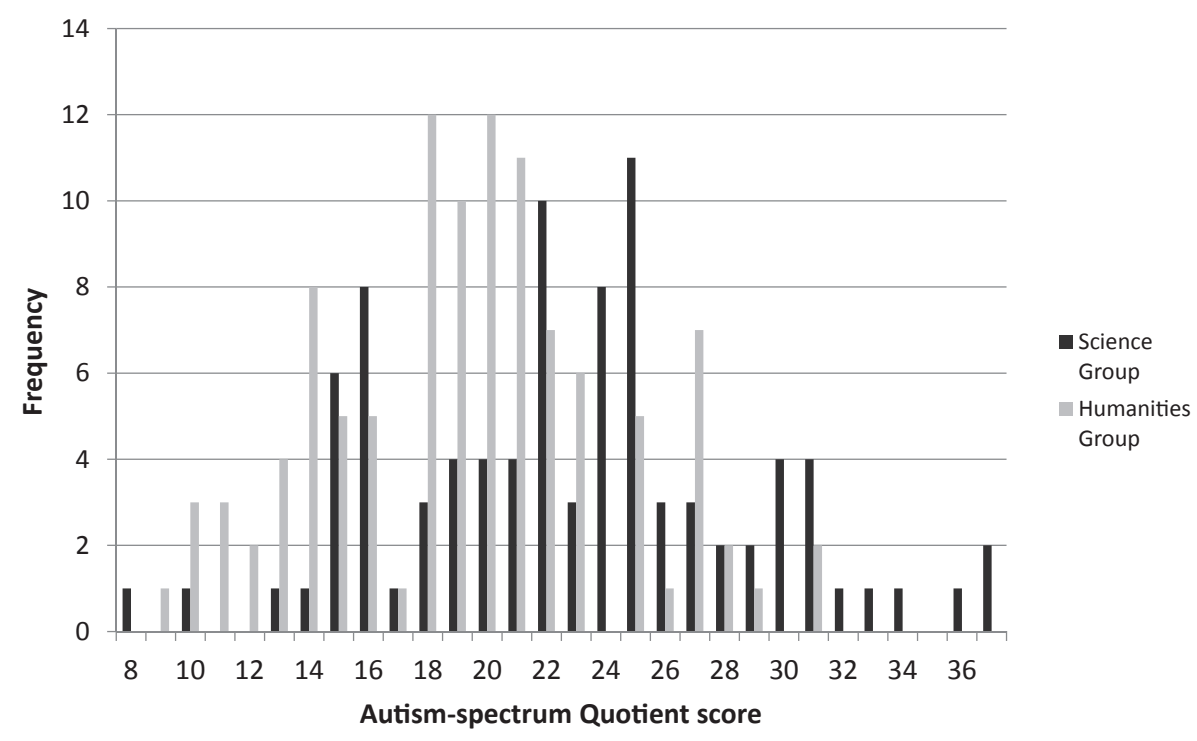

Figure 3: Frequency of obtained scores in the Autism-spectrum Quotient by the Science group and the Humanities group

difficult to imagine what it would be like to be someone else". These items were distributed across the categories of "social skills", "communication skills", and "imagination skills". The complete list is given in Appendix 2. Our results show a positive relationship between participants' score for these thirteen Theory-of-Mind items (a high score implies low Theory-ofMind skills) and the number of logic answers for underinformative items $(r=.165, p=.02)$. Again, such correlation is not shown when considering only students of the science group (Pearson's $r=-.069, N=90, p=.52$ ), but remains significant when considering only students in the humanities group (Pearson's $r=.322, N=108, p=.001$ ). In contrast, there was no relationship between the score for the other 47 items and number of logical responses $(r=.086, p=.226)$.

\section{General discussion}

In Experiments 1 and 2 we found that science students gave less pragmatic answers and were more likely to respond logically to scalar-implicature statements than humanities students. These findings are consistent with 
the observation of higher incidence of autistic traits among science majors compared to humanities majors (Baron-Cohen 1997a; Baron-Cohen et al. 1997; Baron-Cohen 1998; Baron-Cohen et al. 2001). Experiment 2 further showed a positive relationship between typical adults' scores on the Autism-spectrum Quotient, which measures autistic traits, and the number of logical (as opposed to pragmatic) responses to scalar-implicature statements. Finally, there was a trend for lower scores in questions most relevant to the assessment of Theory of Mind to be associated with a higher number of logical responses.

Our results suggest that autistic traits are associated with weaker tendencies to draw pragmatic implicatures. In that respect, our findings are consistent with results of a recent ERP study on autism and pragmatic processing that examined electrophysiological responses to evaluate individual differences in the pragmatic processing of underinformative statements (Nieuwland et al. 2010). They found individual variations in N400 responses based on participants' pragmatic skills, assessed with the Autism-spectrum Quotient. That is, participants with higher Autismspectrum Quotient scores (and, particularly, with higher autistic traits in the Communication subscale) were less sensitive to pragmatic violations in underinformative sentences and actually showed no pragmatic N400 effect. However, there are conflicting results showing no roles of autistic traits but, instead, a role of working memory capacity (Antoniou et al. 2016) or of response times (Heyman \& Schaeken 2015).

Our results are also surprising if one considers the outcomes of previous studies showing intact performance of teens and adults with ASD in scalar-implicature computation tasks (Chevallier et al. 2010; Pijnacker et al. 2009). Similar findings were also reported on children with ASD by $\mathrm{Su}$ and $\mathrm{Su}$ (2015). Pijnacker et al. (2009) tested 56 Dutch adults, 28 with ASD (11 participants with high-functioning autism (HFA) and 17 with Asperger syndrome) and 28 matched controls. ${ }^{2}$ All participants completed a Truth-Value Judgment Task on two different types of scalar terms: 〈some, all $\rangle$ and $\langle$ or, and $\rangle$. Pijnacker et al. expected more logical answers from the ASD group, due to their Theory-of-Mind deficits. However, they did not find differences in the pragmatic answers of the two groups. There was also a positive correlation between the HFA group's pragmatic answers and their verbal intelligence. On the other hand, according to authors,

${ }^{2}$ The distinction between Asperger syndrome and high functioning autism made by Diagnostic and Statistical Manual of Mental Disorders (American Psychiatric Association 2000) has been now abandoned in the last, fifth edition of the DSM (American Psychiatric Association 2013). 
the verbal-intelligence differences between the two ASD subgroups might be a limitation of this study $\left(\mathrm{HFA}_{\text {verbal-int. }}=109.8\right.$, Aspergers verbal-int. $=$ 122.4). Furthermore, authors wrote that it is plausible that Theory of Mind is involved in scalar implicatures computation; however, the study did not assess Theory-of-Mind abilities.

Chevallier et al. (2010) tested 22 ASD adolescents (6 HFA and 16 Aspergers) and 22 controls on the pragmatic interpretation of underinformative connectives; also in their study they did not assess Theory-of-Mind skills. Like Pijnacker et al.'s (2009) findings, the rate of pragmatic interpretations was the same for the ASD and control groups, and there was a positive correlation between pragmatic responses and verbal intelligence, but only in the ASD group. Finally, Su and Su (2015) tested 28 Mandarinspeaking children (age range 4-15 years old) with ASD (12 children with autistic disorder, 15 children with Asperger syndrome and 1 child with pervasive developmental disorder) and 28 controls on the computation of underinformative sentences with the logical words some and every ... or. ASD children did not show any delay in the derivation of scalar implicatures compared to the matched controls.

One possibility to explain the inconsistency of our results with previous findings in literature on autism is that students of scientific disciplines are trained to focus on the literal meaning when performing academic tasks or logic puzzles and this shaped their pragmatic reasoning in our task. This explanation is supported by the fact that scores in the AQ, and particularly of the items we considered related to Theory-of-Mind skills, correlated with the number of pragmatic answers only in the humanities group and not in the scientific group. If, at first glance, this result seemed in contrast with our first hypothesis, we should nonetheless consider that the main goal of our study was precisely to demonstrate that higher autistic traits might lead to more logic answers. If the idea of testing scientific students for such purpose may have been relevant in the light of previous studies (Baron-Cohen 1997a; Baron-Cohen et al. 1997; Baron-Cohen 1998; Baron-Cohen et al. 2001), we now have to consider that the fact that students of scientific disciplines are trained to answer logically in academic tests, might have leveled the differences, in the implicature task, between participants with higher AQ scores and participants with lower AQ scores. On the other hand, our results remain interesting in terms of correlation between autistic traits and pragmatic answers, with less pragmatic answers related to higher autistic traits. 
The inconsistency between current and previous results might also be explained by considering task differences between our study and the others, since it has been demonstrated that, when assessing the understanding or computation of scalar implicatures, sentence-evaluation tasks are preferable to truth-value judgment tasks. Indeed, if we consider the sentence Some dogs are animals, we cannot judge a logic answer (i.e., true) as incorrect, as it is judged in the truth-value judgment task; we should be simply interested in a felicity evaluation, which is whether the participant agrees or disagrees with the content of the statement. Indeed, task sensitivities have been recently demonstrated also with children in the autistic spectrum (Schaeken et al. 2018).

Moreover, people in the autistic spectrum are aware of their pragmatic difficulties and might use compensatory strategies to deal with their deficit, as suggested by Hochstein et al. (2018). This may account in part for the divergence between the findings of previous studies on scalar implicatures in ASD patients and the findings of the present study. Finally, by testing typically developing students with a high Autism-spectrum Quotient, we could assess a high number of participants, whereas previous studies on populations with autism were carried out using smaller samples and it is not yet confirmed whether the same results would also be found using larger samples. Further research is, however, needed to fully explain the origins of the inconsistency in available evidence and clarify the roles of Theory-of-Mind abilities in scalar-implicature computation both in children and adults.

\section{Appendix 1: Statements presented in the Sentence Evaluation Task}

\section{All true}

All snakes are reptiles

All cats are animals

All men are humans

All birds are animals

All cobras are snakes

All dogs are animals

All horses are mammals

All sunflowers are flowers

\section{All false}

All animals are carnivorous

All cats are dogs

All stones are singers

All flowers are professors

All pens are animals 
All children are grandmothers

All televisions are cars

All books are drinks

\section{Some true}

Some dogs are Labrador

Some children are blonde

Some flowers are red

Some cats are Persian

Some houses are rented

Some mobiles are iPhones

Some dresses are blue

Some lakes are big

\section{Some underinformative}

Some children are humans

Some salmons are fish

Some horses are animals

Some tulips are flowers

Some dogs are animals

Some women are humans

Some giraffes are animals

Some roses are flowers

\section{Appendix 2: Items from the Autism-spectrum Quotient that are related to Theory of Mind}

I find it easy to "read between the lines" when someone is talking to me.

I know how to tell if someone listening to me is getting bored.

I find it easy to work out what someone is thinking or feeling just by looking at their face. (Social skills)

I am good at social chit-chat.

When I was young, I used to enjoy playing games involving pretending with other children.

I find it very easy to play games with children that involve pretending.

Other people frequently tell me that what I've said is impolite, even though I think it is polite.

When I'm reading a story, I find it difficult to work out the characters' intentions.

I don' t particularly enjoy reading fiction.

I frequently find that I don't know how to keep a conversation going.

When I talk on the phone, I'm not sure when it's my turn to speak.

I am often the last to understand the point of a joke.

I find it difficult to work out people's intentions. 


\section{References}

Andrés-Roqueta, Clara and Napoleon Katsos. 2017. The contribution of grammar, vocabulary and theory of mind in pragmatic language competence in children with autistic spectrum disorders. Frontiers in Psychology 8. 996.

American Psychiatric Association. 2000. Diagnostic and statistical manual of mental disorders. Fourth edition. Text revision (DSM-IV-TR). Washington, DC: American Psychiatric Association (APA).

American Psychiatric Association. 2013. Diagnostic and statistical manual of mental disorders (5th ed.). Washington, DC: American Psychiatric Association (APA).

Antoniou, Kyriakos, Chris Cummins and Napoleon Katsos. 2016. Why only some adults reject under-informative utterances. Journal of Pragmatics 99. 78-95.

Baron-Cohen, Simon. 1988. Social and pragmatic deficits in autism: Cognitive or affective? Journal of Autism and Developmental Disorders 18. 379-402.

Baron-Cohen, Simon. 1989. Are autistic children "behaviorists"? An examination of their mental-physical and appearance-reality distinctions. Journal of Autism and Developmental Disorders 19. 579-600.

Baron-Cohen, Simon. 1997a. Are children with autism superior at folk physics? New Directions for Child Development 75. 45-54.

Baron-Cohen, Simon. 1997b. Hey! It was just a joke! Understanding propositions and propositional attitudes by normally developing children and children with autism. Israel Journal of Psychiatry and Related Sciences 34. 174-178.

Baron-Cohen, Simon. 1998. Does autism occur more often in families of physicists, engineers, and mathematicians? Autism 2. 296-301.

Baron-Cohen, Simon, Alan M. Leslie and Uta Frith. 1986. Mechanical, behavioural and intentional understanding of picture stories in autistic children. British Journal of Developmental Psychology 4. 113-125.

Baron-Cohen, Simon, Allan M. Leslie and Uta Frith. 1985. Does the autistic child have a 'theory of mind'? Cognition 21. 37-46.

Baron-Cohen, Simon, Sally Wheelwright, Carol Scott, Patrick Bolton and Ian Goodyer. 1997. Is there a link between engineering and autism? Autism 1. 101-109.

Baron-Cohen, Simon, Sally Wheelwright, Richard Skinner, Joanne Martin and Emma Clubley. 2001. The autism-spectrum quotient (AQ): Evidence from asperger syndrome/high-functioning autism, males and females, scientists and mathematicians. Journal of Autism and Developmental Disorders 31. 5-17.

Bott, Lewis and Ira A. Noveck. 2004. Some utterances are underinformative: The onset and time course of scalar inferences. Journal of Memory and Language 51. 437-457.

Capps, Lisa, Jennifer Kehres and Marian Sigman. 1998. Conversational abilities among children with autism and children with developmental delays. Autism 2. 325-344.

Chemla, Emmanuel and Raj Singh. 2014a. Remarks on the experimental turn in the study of scalar implicature. Part I. Language and Linguistics Compass 8. 373-386.

Chemla, Emmanuel and Raj Singh. 2014b. Remarks on the experimental turn in the study of scalar implicature. Part II. Language and Linguistics Compass 8. 387-399.

Chevallier, Coralie, Deirdre Wilson, Francesca Happé and Ira Noveck. 2010. Scalar inferences in autism spectrum disorders. Journal of Autism and Developmental Disorders 40. 1104-1117. 
Chierchia, Gennaro. 2013. Logic in grammar: Polarity, free choice, and intervention. Oxford: Oxford University Press.

Foppolo, Francesca, Maria Terese Guasti and Gennaro Chierchia. 2012. Scalar implicatures in child language: Give children a chance. Language Learning and Development 8. 365-394.

Frith, Uta, John Morton and Alan M. Leslie. 1991. The cognitive basis of a biological disorder: autism. Trends in Neurosciences 14. 433-438.

Grice, H. Paul. 1975. Logic and conversation. In P. Cole and J. L. Morgan (eds.) Syntax and semantics, vol. 3: Speech acts. New York: Academic Press. 41-58.

Guasti, Maria Teresa, Gennaro Chierchia, Stephen Crain, Francesca Foppolo, Andrea Gualmini and Luisa Meroni. 2005. Why children and adults sometimes (but not always) compute implicatures. Language and Cognitive Processes 20. 667-696.

Happé, Francesca. 1993. Communicative competence and theory of mind in autism: A test of relevance theory. Cognition 48. 101-119.

Heyman, Tom and Walter Schaeken. 2015. Some differences in some: Examining variability in the interpretation of scalars using latent class analysis. Psychologica Belgica 55. $1-18$.

Hochstein, Lara, Alan Bale and David Barner. 2018. Scalar implicature in absence of epistemic reasoning? The case of autism spectrum disorder. Language Learning and Development 14. 224-240.

Horn, Larry. 1972. On the semantic properties of logical operators in English. Doctoral dissertation. UCLA.

Jolliffe, Therese and Simon Baron-Cohen. 1997. Are people with autism and Asperger syndrome faster than normal on the Embedded Figures Test? Journal of Child Psychology and Psychiatry 38. 527-534.

Katsos, Napoleon et al. 2016. Cross-linguistic patterns in the acquisition of quantifiers. Proceedings of the National Academy of Sciences 113. 9244-9249.

Leekam, Susan R. and Josef Perner. 1991. Does the autistic child have a metarepresentational deficit? Cognition 40. 203-218.

Leslie, Allan M. and L. Thaiss. 1992. Domain specificity in conceptual development: Neuropsychological evidence from autism. Cognition 43. 225-251.

MacKay, Gilbert and Adrienne Shaw. 2004. A comparative study of figurative language in children with autistic spectrum disorders. Child Language Teaching and Therapy 20. 13-32.

Nieuwland, Mante S., Tali Ditman and Gina R. Kuperberg. 2010. On the incrementality of pragmatic processing: An ERP investigation of informativeness and pragmatic abilities. Journal of Memory and Language 63. 324-346.

Noveck, Ira A. 2001. When children are more logical than adults: Experimental investigations of scalar implicature. Cognition 78. 165-188.

Pijnacker, Judith, Peter Hagoort, Jan Buitelaar, Jan-Pieter Teunisse and Bart Geurts. 2009. Pragmatic inferences in high-functioning adults with autism and Asperger syndrome. Journal of Autism and Developmental Disorders 39. 607-618.

Pouscoulous, Nausicaa, Ira A. Noveck, Guy Politzer and Anne Bastide. 2007. A developmental investigation of processing costs in implicature production. Language Acquisition 14. 347-375. 
Premack, David and Guy Woodruff. 1978. Does the chimpanzee have a theory of mind? Behavioral and Brain Sciences 4. 515-526.

Reddy, Vasudevi, Emma Williams and Amy Vaughan. 2002. Sharing humour and laughter in autism and Down's syndrome. British Journal of Psychology 93. 219-242.

Ruta, Liliana, Domenico Mazzone, Luigi Mazzone, Sally Wheelwright and Simon BaronCohen. 2012. The Autism-Spectrum Quotient - Italian version: A cross-cultural confirmation of the broader autism phenotype. Journal of Autism and Developmental Disorders 42. 625-633.

Schaeken, Walter, Marie van Haeren and Valentina Bambini. 2018. The understanding of scalar implicatures in children with autism spectrum disorder: Dichotomized responses to violations of informativeness. Frontiers in Psychology 9. 1266.

Sigman, Marian, Judy Ungerer, Peter Mundy and Tracy Sherman. 1987. Cognition in autistic children. In D. J. Cohen and R. Paul (eds.) Handbook of autism and pervasive developmental disorders. New York: Wiley. 103-120.

$\mathrm{Su}$, Yi Esther and Lin-Yan Su. 2015. Interpretation of logical words in Mandarin-speaking children with autism spectrum disorders: Uncovering knowledge of semantics and pragmatics. Journal of Autism and Developmental Disorders 45. 1938-1950.

Surian, Luca, Simon Baron-Cohen and Heather van der Lely. 1996. Are children with autism deaf to Gricean maxims? Cognitive Neuropsychiatry 1. 55-72. 